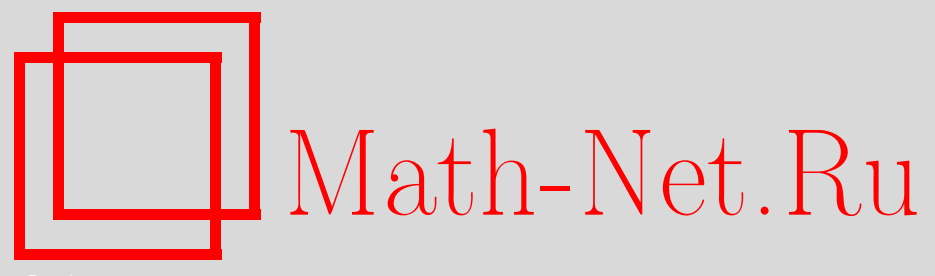

А. В. Бабаш, Периодически внешне наследственные перестановочные автоматы, Дискрет. матем., 2005, том 17, выпуск 3, 68-79

DOI: https://doi.org/10.4213/dm116

Использование Общероссийского математического портала Math-Net.Ru подразумевает, что вы прочитали и согласны с пользовательским соглашением http://www . mathnet.ru/rus/agreement

Параметры загрузки:

IP: 54.80 .97 .219

26 апреля 2023 г., 15:19:01 
УдК 519.7

\title{
Периодически внешне наследственные перестановочные автоматы
}

\author{
(c) 2005 г. A. В. Бабаш
}

\begin{abstract}
Предложены алгоритмы, проверяющие следующее свойство заданного конечного перестановочного автомата: для любой периодической входной последовательности и любого начального состояния период соответствующей выходной последовательности кратен периоду входной последовательности. Оценены параметры сложности таких алгоритмов.
\end{abstract}

Пусть $A=\left(X, S, Y,\left(h_{x}\right)_{x \in X},\left(f_{x}\right)_{x \in X}\right)$ - конечный автомат с входным алфавитом $X$, множеством состояний $S$, выходным алфавитом $Y$, частичными функциями перехода $\left(h_{x}\right)_{x \in X}$ и частичными функциями выхода $\left(f_{x}\right)_{x \in X}$. Через $A(s, P)\left(A_{M}(s, P)\right)$ обозначим выходную последовательность автомата $A$ (последовательность его состояний), отвечающую входной последовательности $P$ и начальному состоянию $s \in S$. Напомним ряд необходимых нам определений (см., например, [1]).

Определение 1. Состояние $s \in S$ автомата $A$ называется состоянием с $L$-потерей информации, если существуют различные входные слова

$$
x(1), x(2), \ldots, x(L), \quad x^{\prime}(1), x^{\prime}(2), \ldots, x^{\prime}(L), \quad x(j), x^{\prime}(j) \in X, \quad j=1, \ldots, L,
$$

длины $L=L_{s}$ автомата $A$, для которых выполнены соотношения

$$
\begin{aligned}
h_{x(L)} h_{x(L-1)} \ldots h_{x(1)} s & =h_{x^{\prime}(L)} h_{x^{\prime}(L-1)} \ldots h_{x^{\prime}(1)} s, \\
f_{x(1)} s & =f_{x^{\prime}(1)} s \\
f_{x(2)} h_{x(1)} s & =f_{x^{\prime}(2)} h_{x^{\prime}(1)} s \\
\ldots & \\
f_{x(L)} h_{x(L-1)} \ldots h_{x(1)} s & =f_{x^{\prime}(L)} h_{x^{\prime}(L-1)} \ldots h_{x^{\prime}(1)} s
\end{aligned}
$$

и не существует различных входных слова длины, меньшей $L_{s}$, с тем же условием. Если состояние автомата не является состоянием с $L$-потерей информации ни для какого конечного $L$, то будем говорить, что $s$ есть состояние без потери информации, в противном случае оно называется состоянием с потерей информации.

В графе переходов автомата $A$ состояние с $L$-потерей информации характеризуется 
существованием подграфа вида
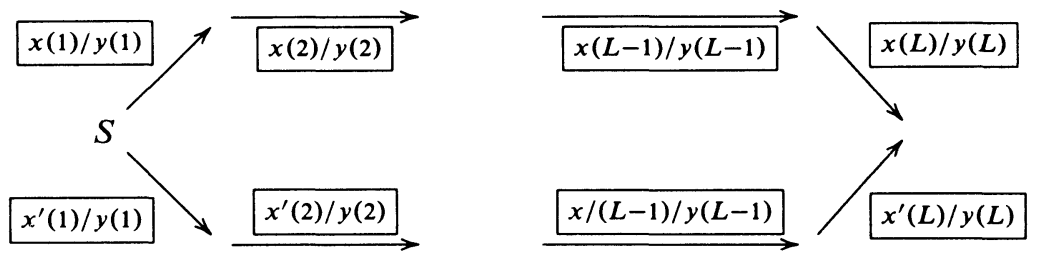

Определение 2. Автомат $A$ называется автоматом без потери информации, если любое его состояние является состоянием без потери информации. В противном случае автомат $A$ называется автоматом с $L$-потерей информации, $L=L_{A}$ совпадает с минимальным $L_{s}$, где минимум берется по всем состояниям $s \in S$ с потерей информации.

Обозначим через $N_{y}, y \in Y$, мощность множества тех состояний $s \in S$ автомата $A$, для которых сушествует элемент $x \in X, x=x(s)$, такой, что $f_{x} s=y$. Через $|S|$ обозначим мощность множества состояний $S$ автомата $A$.

Лемма 1. Пусть $A$-автомат с $L_{A}$-потерей информачии. Тогда

$$
\begin{aligned}
& L_{A} \leqslant 1+\frac{|S|(|S|-1)}{2}, \\
& L_{A} \leqslant 1+\sum_{y \in Y} \frac{N_{y}\left(N_{y}-1\right)}{2} .
\end{aligned}
$$

Доказательство. Пусть $s \in S$ таково, что $L_{s}=L_{A}=L$, а

$$
x(1), x(2), \ldots, x(L), \quad x^{\prime}(1), x^{\prime}(2), \ldots, x^{\prime}(L), \quad x(1) \neq x^{\prime}(1)
$$

- входные слова автомата $A$, для которых выполняются соотношения (1).

Положим

$$
h_{x(j)} h_{x(j-1)} \ldots h_{x(1)} s=s_{j}, \quad h_{x^{\prime}(j)} h_{x^{\prime}(j-1)} \ldots h_{x^{\prime}(1)} s=s_{j}^{\prime}, \quad j=1, \ldots, L-1 .
$$

Очевидно, что $s_{j} \neq s_{j}^{\prime}, j \in\{1, \ldots, L-1\}$. Покажем, что среди неупорядоченных наборов $\left(s_{j} \neq s_{j}^{\prime}\right), j \in\{1, \ldots, L-1\}$, каждый набор встречается не более одного раза. Действительно, предположим, например, что $s_{j(1)}=s_{j(2)}^{\prime}, s_{j(1)}^{\prime}=s_{j(2)}$ для $j(1)<j(2)$. Случай, когда $s_{j(1)}=s_{j(2)}, s_{j(1)}^{\prime}=s_{j(2)}^{\prime}$ рассматривается аналогично.

Очевидно, что для входных слов

$$
\begin{aligned}
& x(1), x(2), \ldots, x(j(1)), x^{\prime}(j(2)+1), \ldots, x^{\prime}(L), \\
& x^{\prime}(1), x^{\prime}(2), \ldots, x^{\prime}(j(1)), x(j(2)+1), \ldots, x(L), \quad x(1) \neq x^{\prime}(1),
\end{aligned}
$$

справедливы равенства

$$
\begin{aligned}
& f_{x(1)} s=f_{x^{\prime}(1)} s \\
& f_{x(j(1))} h_{x(j(1)-1)} \ldots h_{x(1)} s=f_{x^{\prime}(j(1))} h_{x^{\prime}(j(1)-1)} \ldots h_{x^{\prime}(1)} s \\
& f_{x^{\prime}(j(2)+1)} h_{x(j(1))} h_{x(j(1)-1)} \ldots h_{x(1)} s=f_{x(j(2)+1)} h_{x^{\prime}(j(1))} h_{x^{\prime}(j(1))-1} \ldots h_{x^{\prime}(1)} s, \\
& f_{x^{\prime}(L)} h_{x^{\prime}(L-1)} \ldots h_{x^{\prime}(j(2)+1)} h_{x(j(1))} \ldots h_{x(1)} s=f_{x(L)} h_{x(L-1)} \ldots h_{x(j(2)+1)} h_{x^{\prime}(j(1))} \ldots h_{x^{\prime}(1)} s \text {, } \\
& h_{x^{\prime}(L)} h_{x^{\prime}(L-1) \ldots} h_{x^{\prime}(j(2)+1)} h_{x(j(1))} \ldots h_{x(1)} s=h_{x(L)} h_{x(L-1)} \ldots h_{x(j(2)+1)} h_{x^{\prime}(j(1))} \ldots h_{x^{\prime}(1)} s \text {. }
\end{aligned}
$$


Это противоречит предположению о том, что $s$ является состоянием с $L$-потерей информации при $L_{s}=L$. Таким образом, среди наборов неупорядоченных пар состояний $\left(s_{j}, s_{j}^{\prime}\right), j \in\{1, \ldots, L-1\}$, нет одинаковых пар. Отсюда, в частности, можно сделать выводы, что, во-первых,

$$
L=L_{A} \leqslant 1+|S| \frac{|S|-1}{2}
$$

и во-вторых, для любой пары состояний $\left(s_{j}, s_{j}^{\prime}\right)$ существует $x \in X$, при котором $f_{x} s_{j}=f r_{x} s_{j}^{\prime}$, и следовательно, число этих пар не превосходит величины

$$
\sum_{y \in Y} \frac{N_{y}\left(N_{y}-1\right)}{2},
$$

откуда и следует вторая оценка леммы.

Далее предполагается, что изучаемілй автомат $A=\left(X, S, Y,\left(h_{x}\right)_{x \in X},\left(f_{x}\right)_{x \in X}\right)$ является перестановочным автоматом, то есть его частичные функции переходов $\left(h_{x}\right)_{x \in X}$ осуществляют взаимно однозначные преобразования множества $S$.

Если $P=x(1), x(2), \ldots$ - периодическая последовательность элементов входного алфавита $X$ и $s$ - некоторое состояние автомата $A$, то через

$$
A_{M}(s, P)=s, h_{x(1)} s, h_{x(2)} h_{x(1)} s, \ldots
$$

будем обозначать последовательность состояний автомата $A$, отвечающую входной последовательности $P$ и начальному состоянию $s \in S$. Через

$$
A(s, P)=f_{x(1)} s, f_{x(2)} h_{x(1)} s, \ldots
$$

будем обозначать выходную последовательность автомата $A$, отвечающую входной последовательности $P$ и начальному состоянию $s$.

Пусть $\omega(P)-$ период последовательности $P$. Известно, что последовательности $A_{M}(s, P), A(s, P)$ являются периодическими последовательностями (имеется ввиду чисто периодическими), периоды которых являются делителями числа $k(s, P) \omega(P)$, где $k(s, P)$ - минимальное натуральное число $k$, для которого выполнено условие

$$
\left(h_{x(\omega(P))} h_{x(\omega(P)-1)} \ldots h_{x(1)}\right)^{k} s=s .
$$

Другими словами, $k(s, P)$ - длина цикла подстановки $h_{x(\omega(P))} h_{x(\omega(P)-1)} \ldots h_{x(1)}$, содержащей состояние $s$.

Кроме того, если перестановочный автомат $A$ является автоматом Мура, период выходной последовательности $A(s, P)$ делит период последовательности $A_{M}(s, P)$, что в общем случае для перестановочных автоматов Мили не имеет места.

Введем ряд определений.

Определение 3. Перестановочный автомат $A$ называется периодически внешне наследственным (периодически внутренне наследственным), если для любой периодической входной последовательности $P$ и для любого начального состояния $s$ автомата $A$ период выходной последовательности $A(s, P)$ (последовательности состояний $A_{M}(s, P)$ автомата $A)$ кратен периоду входной последовательности $P$. 
Определение 4. Перестановочный автомат $A=\left(X, S, Y,\left(h_{x}\right)_{x \in X},\left(f_{x}\right)_{x \in X}\right)$ называется внешне консервативным, если для любой входной последовательности $P$ периода $\omega(P)$ и любого начального состояния $s \in S$ период выходной последовательности $A(s, P)$ равен $k(s, P) \omega(P)$.

Очевидно, что внешне консервативный автомат $A$ является периодически внешне наследственным. Если, кроме того, $A$ - автомат Мура, то он также и периодически внутренне наследственен. Приводимые ниже примеры показывают, что внешне консервативные автоматы существуют.

Пример 1. Пусть $A=\left(X, S, Y,\left(h_{x}\right)_{x \in X},\left(f_{x}\right)_{x \in X}\right)$ является перестановочным автоматом, где $Y=X \times S$ и $f_{x} s=(x, s),(x, s) \in Y$. Легко проверяется, что данный автомат $A$ является внешне консервативным автоматом.

Пример 2. Пусть перестановочный автомат Мура $A=\left(x, S, Y,\left(h_{x}\right)_{x \in X}, \lambda\right)$ является неавтономным регистром сдвига, например, пусть $X=F_{2}$ - поле из двух элементов, $S=F_{2}^{n}$, для $\left(\varepsilon_{n}, \varepsilon_{n-1}, \ldots, \varepsilon_{1}\right) \in F_{2}^{n}$

$$
\begin{aligned}
& h_{0}\left(\varepsilon_{n}, \varepsilon_{n-1}, \ldots, \varepsilon_{1}\right)=\left(\varphi\left(\left(\varepsilon_{n}, \varepsilon_{n-1}, \ldots, \varepsilon_{1}\right), \varepsilon_{n}, \varepsilon_{n-1}, \ldots, \varepsilon_{2}\right),\right. \\
& h_{1}\left(\varepsilon_{n}, \varepsilon_{n-1}, \ldots, \varepsilon_{1}\right)=\left(\varphi\left(\left(\varepsilon_{n}, \varepsilon_{n-1}, \ldots, \varepsilon_{1}\right)+1, \varepsilon_{n}, \varepsilon_{n-1}, \ldots, \varepsilon_{2}\right),\right.
\end{aligned}
$$

где + обозначает сложение по модулю $2, \varphi\left(z_{n}, z_{n-1}, \ldots, z_{1}\right)$ - двоичная функция вида

$$
\varphi\left(z_{n}, z_{n-1}, \ldots, z_{1}\right)=\Phi\left(z_{n}, z_{n-1}, \ldots, z_{2}\right)+z_{1}, \quad \Phi: F_{2}^{n} \rightarrow F_{2},
$$

и $\lambda\left(\varepsilon_{n}, \varepsilon_{n-1}, \ldots, \varepsilon_{1}\right)=\varepsilon_{1}$.

Легко проверить, что этот автомат внешне консервативен.

Замечание 1. Пусть $A=A_{1} \rightarrow A_{2} \rightarrow \ldots \rightarrow A_{k}$ - последовательное соединение автоматов $A_{1}, \ldots, A_{k}$. Легко видеть, что если автоматы $A_{1}, \ldots, A_{k}$ внешне консервативны (периодически внешне наследственные), то и автомат $A$ внешне консервативен (периодически внешне наследственен).

Из замечания 1 видно, какую важную роль в вопросах синтеза автоматов с гарантированным периодом выходной последовательности играют внешне консервативные и периодически внешне наследственные автоматы. Однако, из их определения не ясно даже, можно ли за конечное число шагов определить, является ли данный автомат внешне консервативным (периодически внешне наследственным). Ранее В. А. Башевым был получен критерий периодически внутренней наследственности автомата, из которого, в частности, следует существование алгоритма для проверки периодически внутренней наследственности автомата. Для перестановочных автоматов критерий формулируется следующим образом: для любой периодической входной последовательности $P$ периода $\omega(P)$ перестановочного автомата $A=\left(X, S, Y,\left(h_{x}\right)_{x \in X},\left(f_{x}\right)_{x \in X}\right)$ и любого его начального состояния $s \in S$ период последовательности состояний $A_{M}(s, P)$, отвечающей $P$ и $s$, кратен $\omega(P)$ тогда и только тогда, когда $h_{x} s \neq h_{x^{\prime}} s$ при любых различных $x, x^{\prime} \in X$ и любом $s \in S$.

Вопрос о существовании алгоритмов для определения внешней наследственности (а также сильной консервативности) до сих пор оставался открытым. В настоящей работе показывается существование указанных алгоритмов и даются верхние оценки параметров их сложности. 
Лемма 2. Для перестановочного автомата $A=\left(X, S, Y,\left(h_{x}\right)_{x \in X},\left(f_{x}\right)_{x \in X}\right)$ c $L_{A}$-nотерей информачии существуют входная последовательность $P$ периода

$$
\omega(P) \leqslant 2\left(\min \left(\frac{|S|(|S|-1)}{2}, \sum_{y \in Y} \frac{N_{y}\left(N_{y}-1\right)}{2}\right)+|S|\right)
$$

и состояние $s$, для которых период выходной последовательности $A(s, P)$ не кратен $\omega(P)$.

Доказательство. По условию леммы найдется состояние $s$ автомата $A$ с $L$-потерей информация, где $L=L_{s}=L_{A}$, в частности, можно указать два входных слова $x(1), x(2), \ldots, x(L)$ и $x^{\prime}(1), x^{\prime}(2), \ldots, x^{\prime}(L)$, для которых

$$
\begin{aligned}
f_{x(1)} s & =f_{x^{\prime}(1)} s=y_{1}, \\
f_{x(2)} h_{x(1)} s & =f_{x^{\prime}(2)} h_{x^{\prime}(1)} s=y_{2}, \\
\ldots & \\
f_{x(L)} h_{x(L-1)} \ldots h_{x(1)} s & =f_{x^{\prime}(L)} h_{x^{\prime}(L-1)} \ldots h_{x^{\prime}(1)} s=y_{L}, \\
h_{x(L)} h_{x(L-1)} \ldots h_{x(1)} s & =h_{x^{\prime}(L)} h_{x^{\prime}(L-1)} \ldots h_{x^{\prime}(1)} s=s^{\prime},
\end{aligned}
$$

где $y_{j} \in Y, j \in\{1, \ldots, L\}$, причем $x(1) \neq x^{\prime}(1)$.

Выберем в графе переходов автомата $A$ путь, ведущий из состояния $s^{\prime}$ в $s$. Это можно сделать в силу перестановочности автомата $A$. Пусть это будет путь, отвечающий последовательности состояний

$$
s^{\prime}, h_{x^{\prime \prime}(1)} s^{\prime}, h_{x^{\prime \prime}(2)} h_{x^{\prime \prime}(1)} s^{\prime}, \ldots, h_{x^{\prime \prime}(m)} \ldots h_{x^{\prime \prime}(1)} s^{\prime}
$$

входной последовательности $x^{\prime \prime}(1), x^{\prime \prime}(2), \ldots, x^{\prime \prime}(m)$ и выходной последовательности $y_{1}^{\prime \prime}, y_{2}^{\prime \prime}, \ldots, y_{m}^{\prime \prime}$. Очевидно, можно считать, что $m \leqslant|S|-1$. Рассмотрим периодическую последовательность $P=z(1), z(2), z(3), \ldots$, где $z(k)=z(k+2(L+m)), k \in\{1,2, \ldots\}$ и

$$
\begin{aligned}
z(t) & =x(t), & & 1 \leqslant t \leqslant L, \\
z(t+L) & =x^{\prime \prime}(t), & & 1 \leqslant t \leqslant m, \\
z(t+L+m) & =x^{\prime}(t), & & 1 \leqslant t \leqslant L, \\
z(t+2 L+m) & =x^{\prime \prime}(t), & & 1 \leqslant t \leqslant m .
\end{aligned}
$$

Тогда последовательность $A(s, P)$ имеет вид

$$
A(s, P)=y_{1}, y_{2}, \ldots, y_{L}, y_{1}^{\prime \prime}, y_{2}^{\prime \prime}, \ldots, y_{m}^{\prime \prime}, y_{1}, y_{2}, \ldots, y_{L}, y_{2}^{\prime \prime}, \ldots, y_{m}^{\prime \prime}, \ldots
$$

и ее период делит $m+L$. Если бы этот период был кратен периоду $\omega(P)$ последовательности $P$, то $m+L$ делилось бы на $\omega(P)$, откуда, в частности, следовало бы, что $z(1)=z(m+L+1)$, то есть $x(1)=x^{\prime}(1)$, что невозможно в силу выбора последовательностей $x(1), x(2), \ldots, x(L)$ и $x^{\prime}(1), x^{\prime}(2), \ldots, x^{\prime}(L)$. Согласно лемме 1

$$
L=L_{A} \leqslant 1+\min \left(\frac{|S|(|S|-1)}{2}, \sum_{y \in Y} \frac{N_{y}\left(N_{y}-1\right)}{2}\right),
$$


поэтому для периода $\omega(P)$ построенной последовательности $P$ справедливо неравенство

$$
\omega(P) \leqslant 2\left(\min \left(\frac{|S|(|S|-1)}{2}, \sum_{y \in Y} \frac{N_{y}\left(N_{y}-1\right)}{2}\right)+|S|\right) .
$$

Замечание 2. Лемма 2 показывает, что периодически внешне наследственный автомат всегда является автоматом без потери информации. Обратное утверждение, вообще говоря, несправедливо. Действительно, рассмотрим автомат Мура

$$
A=\left(X, S, Y,\left(h_{x}\right)_{x \in X},\left(f_{x}\right)_{x \in X}\right),
$$

где $X=\{0,1\}, S=\{1,2,3,4\}, Y=\{0,1\}$, а $h_{0}, h_{1}$ - подстановки,

$$
\begin{aligned}
h_{0} & =\left(\begin{array}{llll}
1 & 2 & 3 & 4 \\
2 & 3 & 4 & 1
\end{array}\right), & h_{1} & =\left(\begin{array}{llll}
1 & 2 & 3 & 4 \\
4 & 1 & 2 & 3
\end{array}\right), \\
\lambda(1) & =\lambda(2)=0, & \lambda(3) & =\lambda(4)=1 .
\end{aligned}
$$

Граф переходов автомата $A$ имеет вид

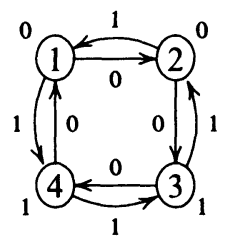

Очевидно, что данный автомат является перестановочным, и непосредственная проверка показывает, что он без потери информации. Тем не менее, для входной последовательности $X=0,1,0,1,0,1, \ldots$ периода $\omega(P)=2$ и начального состояния $s=1$ период выходной последовательности $A(s, P)$ равен 1 и, таким образом, не кратен $\omega(P)$.

Для автомата $A=\left(X, S, Y,\left(h_{x}\right)_{x \in X},\left(f_{x}\right)_{x \in X}\right),|X| \geqslant 2,|Y| \geqslant 2$, выберем подходящую нумерацию элементов выходного алфавита $Y$, при которой

$$
N_{y^{(1)}} \geqslant N_{y^{(2)}} \geqslant \ldots \geqslant N_{y^{(r)}}, \quad Y=\left\{y^{(1)}, y^{(2)}, \ldots, y^{(r)}\right\} .
$$

Заметим, что $N_{y^{(j)}} \leqslant|S|, j=1,2, \ldots, r$, и будем предполагать, что $N_{y^{(r)}} \geqslant 1$, то есть

$$
Y=\bigcup_{x \in X} f_{x}(S)
$$

Лемма 3. Пусть

$$
A=\left(X, S, Y,\left(h_{x}\right)_{x \in X},\left(f_{x}\right)_{x \in X}\right), \quad|X| \geqslant 2, \quad|Y| \geqslant 2, \quad Y=\bigcup_{x \in X} f_{x}(S)
$$

является перестановочным автоматом без потери информачии. Если для любой входной последовательности $P$ периода

$$
\omega(P) \leqslant N_{y^{(2)}}|S|(|S|-1)(|S|-2) \ldots\left(|S|-N_{y^{(2)}}+1\right)
$$

и любого начального состояния $s$ автомата $A$ период выходной последовательности $A(s, P)$ кратен периоду $\omega(P)$ входной последовательности $P$, то автомат $A$ является периодически внешне наследственным. 
Доказательство. Предположим, что выполнено условие леммы 3, но, тем не менее, существуют периодические входные последовательности и состояния, для которых период соответствующих выходных последовательностей не кратен периодам входных последовательностей. Среди них выберем последовательность $P$ периода $\omega(P)=\omega$ и состояние $s_{1} \in S$, для которых величина $k\left(s_{1}, P\right) \omega(P)$ минимальна. Пусть последовательность $P$ имеет вид $P=x(1), x(2), \ldots$, где $x(j)=x(j+\omega)$ для любого $j$. Тогда $A_{M}\left(s_{1}, P\right)=s_{1}, s_{2}, \ldots$, где $s_{j}=h_{x(j-1)} \ldots h_{x(1)} s_{1}, j=2,3, \ldots$ и $A\left(s_{1}, P\right)=y_{1}, y_{2}, \ldots$, где $y_{j}=f_{x(j)} h_{x(j-1)} \ldots h_{x(1)} s_{1}, j=1,2, \ldots$.

Если $W$ - период последовательности $A(s, P)$, то $W$ делит $k\left(s_{1}, P\right) \omega(P)$, то есть для некоторого $L \geqslant 2$ справедливы равенства $k\left(s_{1}, P\right) \omega(P)=k \omega=L W$.

Оценим сверху величину $L W$. Для этого покажем, прежде всего, что для любого $j \in\{1, \ldots, W\}$ состояния $s_{j}, s_{j+W}, \ldots, s_{j+(L-1) W}$ попарно различны. Предположим обратное. Пусть для некоторого $j^{\prime} \in\{1, \ldots, W\}$ справедливо равенство

$$
s_{j^{\prime}+k(1) W}=s_{j^{\prime}+k(2) W}, \quad k(1)<k(2), \quad k(1), k(2) \in\{0,1, \ldots, L-1\} .
$$

Для $P=x(1), x(2), \ldots$ рассмотрим последовательность $P^{j}=x(j), x(j+1), \ldots$ Возможны два случая:

(1) $P^{j^{\prime}+k(1) W}=P^{j^{\prime}+k(2) W}$,

(2) $P^{j^{\prime}+k(1) W} \neq P^{j^{\prime}+k(2) W}$.

Первый случай можно исключить, так как при его выполнении справедливо равенство

$$
\left(P^{j^{\prime}+k(1) W}, s_{j^{\prime}+k(1) W}\right)=\left(P^{j^{\prime}+k(2) W}, s_{j^{\prime}+k(2) W}\right),
$$

что равносильно справедливости равенства

$$
\left(P, s_{1}\right)=\left(P^{k(2)-k(1) W+1}, s_{k(2)-k(1) W+1}\right),
$$

откуда следует, что $(k(2)-k(1)) W \geqslant k \omega$. Последнее неравенство противоречит выбору $k(1), k(2)$.

Во втором случае

$$
\begin{gathered}
A\left(P^{j^{\prime}+k(1) W}, s_{j^{\prime}+k(1) W}\right)=A\left(P^{j^{\prime}+k(2) W}, s_{j^{\prime}+k(2) W}\right), \\
s_{j^{\prime}+k(1) W+k \omega}=s_{j^{\prime}+k(1) W}=s_{j^{\prime}+k(2) W}=s_{j^{\prime}+k(2) W+k \omega},
\end{gathered}
$$

и автомат $A$ оказывается автоматом с $L_{A}$-потерей информации, причем $L_{A} \leqslant k \omega$, что противоречит условию леммы 3. Таким образом, для любого $j \in\{0,1, \ldots, W\}$ состояния $s_{j}, s_{j+W}, \ldots, s_{j+(L-1) W}$ попарно различны. Учитывая теперь равенства

$$
f_{x(j)} s_{j}=f_{x(j+W)} s_{j+W}=\ldots=f_{x(j+(L-1) W)} s_{j+(L-1) W},
$$

получаем, что $L \leqslant N_{y^{(1)}}$. Кроме того, при условии $W \neq 1$ справедливо неравенство $L \leqslant N_{Y^{(2)}}$.

Для получения оценки сверху величины $L W$ остается оценить величину $W$. Покажем, что

$$
W \leqslant 2|S|(|S|-1) \ldots(|S|-L+1) .
$$


Для этого, очевидно, достаточно показать, что при $W \geqslant 3$ среди наборов состояний

$$
\left(\begin{array}{c}
s_{1} \\
s_{1+W} \\
\vdots \\
s_{1+(L-1) W}
\end{array}\right),\left(\begin{array}{c}
s_{2} \\
s_{2+W} \\
\vdots \\
s_{2+(L-1) W}
\end{array}\right), \ldots,\left(\begin{array}{c}
s_{W} \\
s_{2 W} \\
\vdots \\
s_{L W}
\end{array}\right)
$$

каждый набор встречается не более двух раз.

Допустим обратное, без ограничения общности будем считать, что первый набор $s_{1}, s_{1+W}, \ldots, s_{1+(L-1) W}$ встречается по крайней мере три раза, то есть существуют $k(1), k(2) \in\{2,3, \ldots, W\}, k(1)<k(2)$, такие, что

$$
\begin{aligned}
s_{1} & =s_{k(1)}=s_{k(2)}, \\
s_{1+W} & =s_{k(1)+W}=s_{k(2)+W} \\
& \ldots \\
s_{1+(L-1) W} & =s_{k(1)+(L-1) W}=s_{k(2)+(L-1) W} .
\end{aligned}
$$

Построим вспомогательные периодические последовательности $P^{\prime}$ и $P^{\prime \prime}$.

Пусть

$$
P^{\prime}=x^{\prime}(1), x^{\prime}(2), \ldots, x^{\prime}(j), \ldots
$$

где для любого $j$

$$
x^{\prime}(j)=x^{\prime}(j+L(W-k(1)+1))
$$

и начальное слово $x^{\prime}(1), x^{\prime}(2), \ldots, x^{\prime}(L(W-k(1)+1))$ длины $L(W-k(1)+1)$ последовательности $P^{\prime}$ имеет вид

$$
\begin{array}{r}
x(k(1)), x(k(1)+1), \ldots, x(k(2)), x(k(2)+1), \ldots, x(W), x(k(1)+W), \\
x(k(1)+W+1), \ldots, x(k(2)+W), x(k(2)+W+1), \ldots, x(2 W), \ldots, \\
x(k(1)+(L-1) W), x(k(1)+(L-1) W+1), \ldots, x(k(2)+(L-1) W), \\
x(k(2)+(L-1) W+1), \ldots, x(L W) .
\end{array}
$$

Пусть

$$
P^{\prime \prime}=x^{\prime \prime}(1), x^{\prime \prime}(2), \ldots, x^{\prime \prime}(j), \ldots,
$$

где для любого $j$

$$
x^{\prime \prime}(j)=x^{\prime}(j+L(W-k(2)+k(1)))
$$

и начальное слово $x^{\prime \prime}(1), x^{\prime \prime}(2), \ldots, x^{\prime \prime}(L(W-k(1)+1))$ длины $L(W-k(2)+k(1))$ последовательности $P^{\prime \prime}$ имеет вид

$$
\begin{gathered}
x(1), x(2), \ldots, x(k(1)-1), x(k(2)), x(k(2)+1), \ldots, x(W), x(W+1), x(W+2), \ldots, \\
x(k(1)-1+W), x(k(2)+W), x(k(2)+W+1), \ldots, x(2 W), \ldots, x((L-1) W+1), \\
x((L-1) W+2), \ldots, x(k(1)-1+(L-1) W), x(k(2)+(L-1) W), \\
x(k(2)+1+(L-1) W), \ldots, x(L W) .
\end{gathered}
$$

Рассмотрим последовательности $A_{M}\left(s_{1}, P^{\prime}\right), A\left(s_{1}, P^{\prime}\right), A_{M}\left(s_{1}, P^{\prime \prime}\right), A\left(s_{1}, P^{\prime \prime}\right)$. Последовательности $A_{M}\left(s_{1}, P\right)$ запишем в виде

$$
A_{M}\left(s_{1}, P^{\prime}\right)=s_{1}^{\prime}, s_{2}^{\prime}, \ldots, s_{j}^{\prime}, \ldots,
$$


где $s_{j}^{\prime}=s_{j+L(W-k(1)+1)}^{\prime}, j \in\{1,2, \ldots\}$, и начальная часть длины $L(W-k(1)+1)$ последовательности $A_{M}\left(s_{1}, P^{\prime}\right)$ имеет вид

$$
\begin{array}{r}
s_{1}, s_{k(1)+1}, s_{k(1)+2}, \ldots, s_{k(2)}, s_{k(2)+1}, \ldots, s_{W}, s_{1+W}, s_{1+k(1)+W}, s_{2+k(1)+W}, \ldots, s_{k(2)+W} \\
s_{1+k(2)+W}, \ldots, s_{2 W}, \ldots, s_{1+(L-1) W}, s_{1+k(1)+(L-1) W}, s_{2+k(1)+(L-1) W}, \ldots, \\
s_{k(2)+(L-1) W}, s_{k(2)+1+(L-1) W}, \ldots, s_{L W} .
\end{array}
$$

Последовательность $A\left(s_{1}, P^{\prime}\right)$ запишем в виде

$$
A\left(s_{1}, P^{\prime}\right)=y_{1}^{\prime}, y_{2}^{\prime}, \ldots
$$

где $y_{j}^{\prime}=y_{j+W-k(1)+1}^{\prime}$, и начальная часть длины $W-k(1)+1$ последовательности $A\left(s_{1}, P^{\prime}\right)$ имеет вид

$$
y_{k(1)}, y_{k(1)+1}, \ldots, y_{k(2)}, y_{k(2)+1}, \ldots, y_{W}
$$

Легко видеть, что $k\left(s_{1}, P^{\prime}\right) \omega\left(P^{\prime}\right)<k\left(s_{1}, P\right) \omega(P)$. Из условия минимальности числа $k\left(s_{1}, P\right) \omega(P)$ и полученного неравенства следует, что период $W^{\prime}$ выходной последовательности $A\left(s_{1}, P^{\prime}\right)$ кратен $\omega\left(P^{\prime}\right)$. Кроме того, очевидно, что $W^{\prime}$ делит $W-k(1)+1$ и поэтому $\omega\left(P^{\prime}\right)$ делит $W-k(1)+1$, откуда следует, что для $m \in\{k(1), k(2), \ldots, W\}$

$$
x(m)=x(m+W)=\ldots=x(m+(L-1) W) .
$$

Рассмотрим вторую последовательность $P^{\prime \prime}$ и соответствующие ей последовательности $A_{M}\left(s_{1}, P^{\prime \prime}\right), A\left(s_{s}, P^{\prime \prime}\right)$. Аналогично показывается, что выполняются соотношения

$$
x(m)=x(m+W)=\ldots=x(m+(L-1) W), m \in\{1,2, \ldots, k(1)-1\} .
$$

Равенства (2) и (3) означают кратность величины $W$ периоду $\omega$ последовательности $P$. Полученное противоречие доказывает, что

$$
W \leqslant 2|S|(|S|-1) \ldots(|S|-L+1)
$$

откуда при $W>1$ следует, что

$$
L W \leqslant 2 N_{y^{(2)}}|S|(|S|-1) \ldots\left(|S|-N_{y^{(2)}}+1\right)
$$

Кроме того легко видеть, что эта оценка справедлива и для $W=1$. Следовательно,

$$
\omega(P) \leqslant 2 N_{y^{(2)}}|S|(|S|-1) \ldots\left(|S|-N_{y^{(2)}}+1\right) .
$$

Последняя оценка противоречит условию леммы 3. Таким образом, лемма 3 полностью доказана.

Из лемм 2 и 3 вытекает следующая теорема. 
Теорема 1. Перестановочный автомат

$$
A=\left(X, S, Y,\left(h_{x}\right)_{x \in X},\left(f_{x}\right)_{x \in X}\right), \quad|X| \geqslant 2, \quad|Y| \geqslant 2, \quad Y=\bigcup_{x \in X} f_{x}(S),
$$

явлется периодически внешне наследственным автоматом тогда и только тогда, когда для любого начального состояния $s$ автомата $A$ и любой его входной периодической последовательности $P$ периода $\omega(P)$, не превосходящего максимума чисел

$$
2 N_{y^{(2)}}|S|(|S|-1) \ldots\left(|S|-N_{y^{(2)}}+1\right), 2\left(\min \left(\frac{|S|(|S|-1)}{2}, \sum_{y \in Y} \frac{N_{y}\left(N_{y}-1\right)}{2}\right)+|S|\right)
$$

период выходной последовательности $A(s, P)$ кратен $\omega(P)$.

Следствие 1. Существует алгоритм, проверяющий, является ли данный автомат периодически внешне наследственным.

Лемма 4. Пусть перестановочный автомат

$$
A=\left(X, S, Y,\left(h_{x}\right)_{x \in X},\left(f_{x}\right)_{x \in X}\right), \quad|X| \geqslant 2, \quad|Y| \geqslant 2, \quad Y=\bigcup_{x \in X} f_{x}(S),
$$

является автоматом без потери информачии. Если для любой входной периодической последовательности $P$ периода

$$
\omega(P) \leqslant N_{y^{(2)}}|S|(|S|-1) \ldots\left(|S|-N_{y^{(2)}}+1\right)
$$

и любого начального состояния $s$ автомата А период последовательности $A(s, P)$ равен $k(s, P) \omega(P)$, то автомат $A$ является внешне консервативным.

Доказательство. Доказательство леммы 4 почти полностью совпадает с доказательством леммы 3. В обозначениях, примененных при доказательстве леммы 3 , имеющееся отличие доказательства леммы 4 от леммы 3 состоит в том, что среди наборов

$$
\left(\begin{array}{c}
s_{j} \\
s_{j+W} \\
\vdots \\
s_{j+(L-1) W}
\end{array}\right), \quad j=1,2, \ldots, W
$$

каждый набор встречается не более одного раза (в доказательстве леммы 3 - не более двух раз). Покажем это. Пусть, например, $t \in\{2, \ldots, W\}$ и

$$
\begin{aligned}
s_{1} & =s_{t}, \\
s_{1+W} & =s_{t+W}, \\
& \cdots \\
s_{1+(L-1) W} & =s_{t+(L-1) W} .
\end{aligned}
$$

Построим вспомогательную последовательность

$$
P^{\wedge}=x(1), x(2), \ldots, x(j),
$$


где $x(j)=x(j+L(W-t+1))$ для любого $j$ и начальное слово длины $L(W-t+1)$ последовательности $P^{\wedge}$ имеет вид

$$
\begin{aligned}
& x(t), x(t+1), \ldots, x(W), x(t+W), x(t+1+W), \ldots, x(2 W), \\
& \ldots, x(t+(L-1) W), x(t+1+(L-1) W), \ldots, x(L W) .
\end{aligned}
$$

Тогда последовательности $A_{M}\left(s_{1}, P^{\wedge}\right), A\left(s_{1}, P^{\wedge}\right)$ имеют вид

$$
A_{M}\left(s_{1}, P^{\wedge}\right)=s_{1}^{\wedge}, s_{2}^{\wedge}, \ldots,
$$

где $s_{j}^{\wedge}=s_{j}+L(W-t+1)$ для любого $j$ и

$$
\begin{aligned}
& s_{1}^{\wedge}, s_{2}^{\wedge}, \ldots, s_{L(W-t+1)}^{\wedge} \\
& \quad=s_{1}, s_{1+t}, \ldots, s_{W}, s_{1+W}, s_{1+t+W}, \ldots, s_{2 W}, \ldots, s_{1+(L-21) W}, \ldots, s_{L W},
\end{aligned}
$$

а последовательность

$$
A\left(s_{1}, P^{\wedge}\right)=y_{1}^{\wedge}, y_{2}^{\wedge}, \ldots,
$$

где $y_{j}^{\wedge}=y_{j+W-t+1}^{\wedge}$ для любого $j$ и

$$
y_{1}^{\wedge}, y_{2}^{\wedge}, \ldots, y_{W-t+1}^{\wedge}=y_{t}, y_{t+1}, \ldots, y_{W}
$$

Очевидно, что

$$
k\left(s_{1}, P^{\wedge}\right) \omega\left(P^{\wedge}\right)<k\left(s_{1}, P\right) \omega(P) .
$$

Поэтому, согласно предположению, период $W^{\wedge}$ последовательности $A\left(s_{1}, P^{\wedge}\right)$ равен $k\left(s_{1}, P^{\wedge}\right) \omega\left(P^{\wedge}\right)$. Так как $W^{\wedge}$ делит $W-t+1$, то $k\left(s_{1}, P^{\wedge}\right) \omega\left(P^{\wedge}\right)$ делит $W-t+1$.

Если $\tau$ - период последовательности $A_{M}\left(s_{1}, P^{\wedge}\right)$, то $\tau$ делит $k\left(s_{1}, P^{\wedge}\right) \omega\left(P^{\wedge}\right)$. Следовательно, $\tau$ делит $W-t+1$.

Из последнего соотношения вытекает равенство $s_{t}=s_{t+W}$, что при $L>1$ невозможно, как мы убедились ранее. Таким образом, заключаем, что при $L>1$

$$
W \leqslant|S|(|S|-1) \ldots(|S|-L+1) .
$$

Очевидно, что эта оценка справедлива и при $L=1$. В остальном доказательство леммы 4 полностью совпадает с доказательством леммы 3.

Из лемм 2 и 4 непосредственно вытекает теорема 2.

Теорема 2. Перестановочный автомат

$$
A=\left(X, S, Y,\left(h_{x}\right)_{x \in X},\left(f_{x}\right)_{x \in X}\right), \quad|X| \geqslant 2, \quad|Y| \geqslant 2, \quad Y=\bigcup_{x \in X} f_{x}(S),
$$

является внешне консервативным автоматом тогда и только тогда, когда для любого начального состояния $s$ автомата $А$ и любой его входной периодической последовательности $P$ периода $\omega(P)$, не превосходящего максимума чисел

$$
N_{y^{(2)}}|S|(|S|-1) \ldots\left(|S|-N_{y^{(2)}}+1\right), 2\left(\min \left(\frac{|S|(|S|-1)}{2}, \sum_{y \in Y} \frac{N_{y}\left(N_{y}-1\right)}{2}\right)+|S|\right)
$$

период выходной последовательности $A(s, P)$ равен величине $K(s, P) \omega(P)$. 
Следствие 2. Существует алгоритм, проверяющий, является ли данный перестановочный автомат сильно консервативным.

\section{Список литературы}

1. Гилл А., Введение в теорию конечных автоматов. Наука, Москва, 1966.

Статья поступила 05.06.2003. 